Published in final edited form as:

Nature. 2013 January 24; 493(7433): 482-483. doi:10.1038/493482a.

\title{
Strength in numbers
}

\author{
MORGAN SHENG, \\ Department of Neuroscience, Genentech Inc., South San Francisco, California 94080, USA. \\ sheng.morgan@gene.com
}

ROBERTO MALINOW, and

Center for Neural Circuits and Behavior, Division of Biology, Neurobiology Section, and the Department of Neurosciences, University of California, San Diego, La Jolla, California 92093, USA.rmalinow@ucsd.edu

\section{RICHARD HUGANIR}

Department of Neuroscience, Howard Hughes Medical Institute, Johns Hopkins University School of Medicine, Baltimore, Maryland 21205, USA. rhuganir@jhmi.edu

\section{Abstract}

A process called long-term potentiation mediates information storage - learning and memory at the level of neurons. An in vitro study turns the molecular understanding of this process on its head. But researchers' opinions differ as to what can be inferred from these data.

\section{One step forward}

The surprising findings of Granger et al. ${ }^{1}$ invoke a possible shift in our view of how AMPA receptors are recruited to synapses.

The prevailing mechanistic view of LTP has focused on the GluA1 and GluA2 sub units of AMPA receptors, whose cytoplasmic tails (C-tails) dangle inside the cell. A variety of proteins bind differentially to the C-tails of GluA1 and GluA2, and disruption of these protein interactions affect AMPA-receptor distribution and trafficking in neurons ${ }^{2}$. An appealing, albeit unproven, hypothesis is that protein interactions with the C-tails of GluA subunits - interactions that are somehow regulated by synaptic activity — would usher AMPA receptors into synapses to induce LTP.

Indeed, compelling studies from Malinow and co-workers concluded that specifically GluA1 and its C-tail are essential for the delivery of AMPA receptors to synapses during LTP (for a review, see ref. 3). However, these experiments relied largely on overexpression of GluA1 and/or GluA2 in hippocampal neurons in which normal endo genous AMPA receptors were still present and so possibly interfered with trafficking of the exogenous subunits.

In Granger and colleagues' experiments, the genes encoding GluA1, GluA2 and GluA3 were deleted, and so endogenous AMPA receptors were absent. It was in this setting that the authors found that not only were the known protein interactions of the GluA1 C-tail un necessary for LTP, but also GluA2 (previously thought not to support LTP $^{3}$ ) and even kainate receptors were sufficient to mediate LTP.

(C) 2013 Macmillan Publishers Limited. All rights reserved

M.S. declares competing financial interests. See go.nature.com/svmjy6 for details. 
This 'molecular replacement' of GluA subunits in neurons that are devoid of AMPA receptors can be rejected as being just as artificial as the experiments overexpressing them in the presence of endogenous AMPA receptors. Nonetheless, Granger and co-authors' data are intriguing because they show that, at least in the context of neurons lacking AMPA receptors, several different kinds of glutamate receptor can be recruited to synapses and are sufficient to support LTP - irrespective of their C-tails and presumably regardless of their associated proteins and accessory subunits.

This conclusion turns the mechanistic spotlight on the overall structural rearrangements that occur in the synapse during LTP - especially changes to a structure called the post-synaptic density, in which AMPA receptors are clustered ${ }^{4}$ — and how such rearrangements might lead to the capture of AMPA receptors. Indeed, the abundance of scaffolding proteins, such as PSD-95, capable of corralling AMPA receptors in the synapse, is regulated by phosphorylation, and such proteins may have an underestimated role in synaptic plasticity 5,6 - the process by which synapses grow stronger or weaker depending on their activity level.

Even if changes in synapse architecture are the main drivers for trapping AMPA receptors during LTP, it is still difficult to imagine how this mechanism could operate completely independently of the C-tails of the receptors or their accessory subunits. To resolve the roles of the different GluA sub units and their C-tails in LTP, it would be helpful to generate data from more subtle genetic experiments in vivo - in which, for example, the C-tail of endogenous GluA1 is replaced with the C-tail of GluA2, without other wise altering the expression of the proteins.

\section{Two steps back}

In Granger and co-workers ${ }^{1}$ experimental set-up, synapses can recruit any type of glutamate receptor to mediate LTP - a remarkable finding that suggests an incredible versatility for synapses. But does such behaviour occur when synapses contain their normal complement of proteins?

Genetically deleting all AMPA receptors, as the authors have done in their elegant system, may fundamentally change AMPA-receptor trafficking compared with that in normal synapses. Indeed, GluA1 subunits lacking their C-tail could reach synapses only when all AMPA receptors were deleted and not in the more normal situation, in which other AMPAreceptor subunits were present. Our interpretation of this observation is that the C-tail endows GluA1 with a competitive advantage to reach the synapse.

To address the mechanisms underlying LTP at normal synapses, more subtle experimental manipulations may be more informative; these could include introducing mutations ('knockin' mutations) into the genes encoding GluA1 and GluA2 to alter their C-tail structure without affecting their expression level. In fact, previous studies ${ }^{7,8}$ involving such mutations have shown that the $\mathrm{C}$-tail phosphorylation sites and protein-interacting domains of these subunits are important for several forms of synaptic plasticity, including LTP.

Synapses lacking AMPA receptors may behave differently from normal synapses because loss of some essential regulatory proteins makes them less selective. Are AMPA-receptor subunits responsible for maintaining such crucial proteins at the synapse? It could be that synapses without AMPA receptors - such as those that are prevalent early in development ${ }^{9}$ - have lower selectivity for receptors during LTP than do more mature synapses containing AMPA receptors. Alternatively, there may be competition for entering the synapse between different receptors that have different interaction partners at the synapse to control their lifetime there. If that is the case, if a competitor with several such partners, say AMPA 
receptors, is removed, then other receptors with minimal numbers of partners and interactions could enter and remain in the synapse.

Granger and co-authors' study, therefore, may point to a novel view that synapses are sites of competition: how efficiently a receptor enters the synapse depends not only on its structural components, but also on those of its competitors. Such factors may determine how AMPA receptors get into and out of a synapse during conditions of synapse maintenance and plasticity.

But whatever the mechanism involved, the present study will focus future research on the structural changes that occur at the synapse after LTP. Many scaffolding proteins regulate the complex structure of the postsynaptic density, and, according to Granger et al., structural changes may occur that do not require specific glutamate receptor types. However, the identity of these common scaffolding proteins is not obvious, because most such proteins are known to be specific for AMPA or kainate receptors. In fact, some of the same authors have previously shown that AMPA-receptor-associated proteins known as TARPs are crucial for LTP $^{10}$. Yet the present study refutes those data and shows that even kainate receptors, which do not interact with TARPs, can completely rescue LTP in the absence of AMPA receptors.

So, one step forward, two steps back. The search for the mechanism underlying LTP is well into its third decade, and it looks as if it will continue to fascinate and elude neuroscientists for the next decade, too.

\section{References}

1. Granger AJ, Shi Y, Lu W, Cerpas M, Nicoll RA. Nature. 2013; 493:495-500. [PubMed: 23235828]

2. Anggono V, Huganir RL. Curr. Opin. Neurobiol. 2012; 22:461-469. [PubMed: 22217700]

3. Malinow R. Phil. Trans. R. Soc. Lond. B. 2003; 358:707-714. [PubMed: 12740116]

4. Sheng, M.; Kim, E. Cold Spring Harb. Perspect. Biol. 2011. http://dx.doi.org/10.1101/ cshperspect.a005678

5. Opazo P, Sainlos M, Choquet D. Curr. Opin. Neurobiol. 2012; 22:453-460. [PubMed: 22051694]

6. Kim MJ, et al. Neuron. 2007; 56:488-502. [PubMed: 17988632]

7. Lee H-K, et al. Cell. 2003; 112:631-643. [PubMed: 12628184]

8. Steinberg JP, et al. Neuron. 2006; 49:845-860. [PubMed: 16543133]

9. Wu G-Y, Malinow R, Cline HT. Science. 1996; 274:972-976. [PubMed: 8875937]

10. Tomita S, Stein V, Stocker TJ, Nicoll RA, Bredt DS. Neuron. 2005; 45:269-277. [PubMed: 15664178] 


\section{THE PAPER IN BRIEF}

Information storage involves stimulation-induced strengthening of synaptic communication between neurons over a long time period.

For some forms of memory, this phenomenon, called long-term potentiation (LTP), occurs in the brain's hippocampus region and involves AMPA receptors, which are activated by the neurotransmitter glutamate.

It is widely believed that the cytoplasmic tail of GluA1, which is one of four isoforms that can constitute the four-subunit AMPA receptor, is essential for trafficking of the receptor to synapses to mediate LTP (Fig. 1).

Granger et al. ${ }^{1}$ carried out a single-cell-based study to investigate the minimum amino-acid sequence of this tail that is required for LTP.

- They found that the GluA1 cytoplasmic tail was not required for LTP at all.

In fact, the expression of a different glutamate receptor, the kainate receptor, was sufficient for LTP to occur.

The only prerequisite for this process seemed to be a large reserve pool of glutamate receptors that could move to synapses to mediate LTP. 


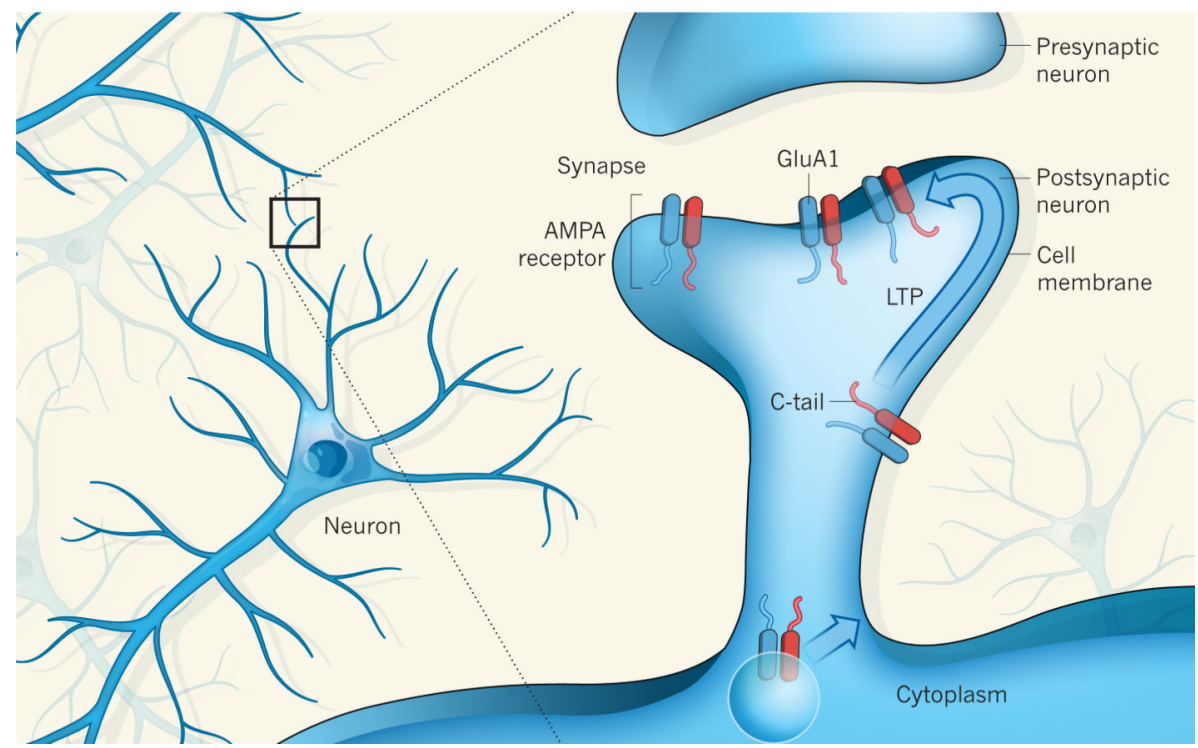

Figure 1. AMPA receptors at the synapse

At the level of neurons, long-term potentiation (LTP) is mediated by AMPA receptors, which are transported from intracellular compartments and elsewhere in the cell membrane to the membrane of the postsynaptic neuron. Surprisingly, Granger et al. ${ }^{1}$ report that the cytoplasmic tail of the GluA1 subunit of AMPA receptors is not required for this trafficking and thus for LTP. Instead, they propose that any type of glutamate receptor can support LTP, as long as enough of them reach the membrane. 